\title{
Automatic detection of end QRS notching or slurring
}

\author{
Elaine N. Clark, MA, ${ }^{\mathrm{a}, \star}$ Ibraheem Katibi, MD, FACC, ${ }^{\mathrm{b}}$ Peter W. Macfarlane, DSc, FRCP ${ }^{\mathrm{a}}$ \\ ${ }^{\mathrm{a}}$ Institute of Cardiovascular and Medical Sciences, University of Glasgow, UK \\ ${ }^{\mathrm{b}}$ Department of Medicine, University of Ilorin, Nigeria \\ http://dx.doi.org/10.1016/j.jelectrocard.2013.10.007
}

\begin{abstract}
The purpose of this study was to define criteria suited to automated detection of end QRS notching and slurring and to evaluate their accuracy. One hundred resting 12 lead ECGs from young adult men, split randomly into equal training and test sets, were examined independently by two reviewers for the presence of such notching or slurring. Consensus was reached by re-examination. Logic was added to the Glasgow resting ECG program to automate the detection of the phenomenon. After training, the automated detection had a sensitivity (SE) of $92.1 \%$ and a specificity (SP) of $96.6 \%$. For the test set, SE was $90.5 \%$, SP $96.5 \%$. Two populations of healthy subjects - one Caucasian, one Nigerian - were analysed using the automated method. The prevalence of notching/slurring with peak/onset amplitude respectively $\geq 0.1 \mathrm{mV}$ in two contiguous inferolateral leads was $23 \%$ and $29 \%$ respectively. In conclusion, the detection of end QRS notching or slurring can be automated with a high degree of accuracy.
\end{abstract}

Keywords: $\quad$ Early repolarization; Automated ECG; Racial differences

* Corresponding author: Elaine.Clark@glasgow.ac.uk

\section{Introduction}

In the last few years, there has been renewed interest in the significance of end QRS notching or slurring. This is one of the characteristics of early repolarization (ER) [1]. It has been reported that the presence of a notch or slur in the downslope of the $\mathrm{R}$ wave together with a horizontal/ descending ST segment is a risk factor for cardiovascular mortality [2,3]. However, there is no clear definition of this morphology and there has been considerable variety in reporting this feature visually. If standard definitions for notching and slurring could be found that are acceptable to all concerned, then they could be incorporated into criteria for diagnostic analysis.

ECGs are commonly reported using computer programs. The aim of this study was to determine if end QRS notching and slurring could be detected using automated methods, and to assess the accuracy of such automated detection of this end $\mathrm{R}$ wave phenomenon by comparing manual and computer detection for a set of ECGs.

\section{Methods}

One hundred resting 12 lead ECGs from young adult men (mean age $24.8 \pm 3.2$ years) were split randomly into equal training and test sets. These were a subset of ECGs from a dataset of 1496 apparently healthy Caucasians (859 males and 637 females with an age range of 18-82 years) collected in the West of Scotland in the 1980s, recorded using a locally designed and built electrocardiograph that recorded 8 seconds of data, with a sampling rate of 500 samples/sec. The subgroup of young adult men was chosen as the prevalence of end QRS notching and slurring is known to be higher in this section of the population [4]. The waveforms for the training set were examined independently by two reviewers for the presence of end QRS notches and slurs (Fig. 1). The tracings used for the review were the average beats for each of the 12 leads. The reviewers marked the leads on the paper printout with an ' $\mathrm{N}$ ' or ' $\mathrm{S}$ ' for notch or slur respectively where appropriate.

The protocol involved marking all notches and slurs in leads II, III, aVF, V4, V5 and V6 unless they were considered too small (as defined later) to be of any significance. A notch was defined as a reversal of slope distinguishable to the eye, and a slur as a change of QRS slope on the terminal component of an $\mathrm{R}$ wave. Where reviewers differed initially, consensus was reached by re-examination.

As a starting point for automating detection, preliminary definitions were set to describe the morphologies in question. While it is common to report notches in automated ECG reports, this is not true of slurs. The definitions were based on ECG wave measurement standards [5] and on the criteria used in recent studies $[1,3,6]$. 

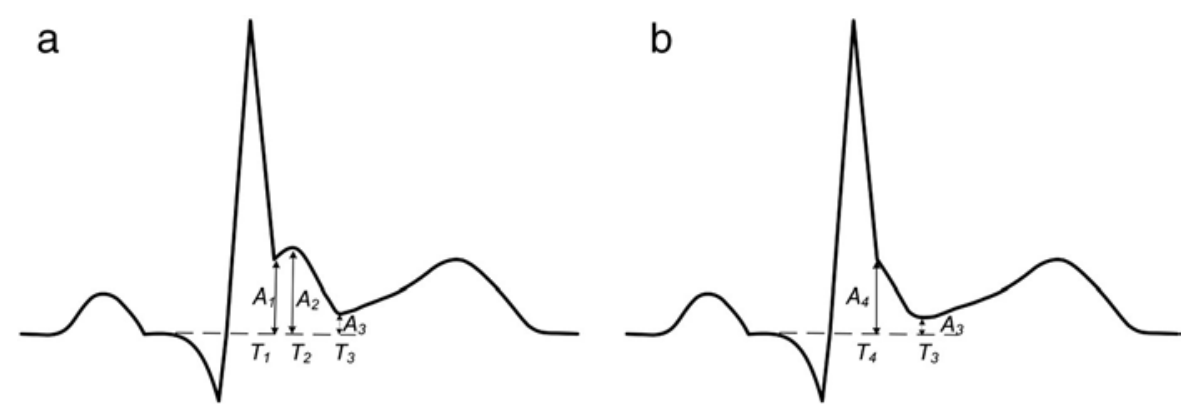

Fig. 1. Measurements of amplitudes (A) and sample numbers (T) for a QRS end notch (1a) or slur (1b).

Various points on the notches and slurs were chosen to be measured (Fig. 1). The following requirements were stipulated: the last component of the QRS complex was an $\mathrm{R}$ wave; the $\mathrm{R}$ wave duration was greater than $40 \mathrm{msec}$; the notch or slur was on the downward slope of the $\mathrm{R}$ wave; the start of the notch or slur was more than $10 \mathrm{msec}$ from the end point of the complex; the notch amplitude A2 or slur amplitude A4 (Fig. 1) had a minimum allowed amplitude of $0.05 \mathrm{mV}$ and maximum of $0.5 \mathrm{mV}$.

The preliminary definitions for notching and slurring were implemented in the wave measurement module of the Glasgow resting ECG program [7]. There is considerable debate in the current literature about the definition of QRS end. The conventional QRS end, i.e. ST onset, is used in the program and is determined from the spatial velocity over all leads and from changes in slopes and amplitudes for each lead. The current method of identifying a notch by finding changes in slope was used and the additional measurements were stored.
New logic was added to identify and measure a slur at the end of the $\mathrm{R}$ wave. The point of inflection was determined by using the same method as is used to identify a delta wave in the WPW pattern, namely finding the inflection point (IP) on the curve (Fig. 2) which minimises the sum of the areas between the curve and the two straight lines drawn from the point of inflection - one connecting to the start of the slope and the other to the end of the slope. The angle of the slope had to be above a minimum value to qualify as a slur.

The category of notch or slur for each lead was output from the program for analysis along with the slope of the ST segment and the ST amplitude at the QRS end point. The slope of a line between the QRS end and the point at 3/8th of the ST-T segment is used as a measurement of ST slope. A slope $\leq 10^{\circ}$ was defined as horizontal or downward sloping.

The manual and automated categorizations were compared and basic statistics obtained, using Excel and IBM SPSS Statistics, for the ECGs in the training set. The classification and comparison were subsequently carried out on the test set.

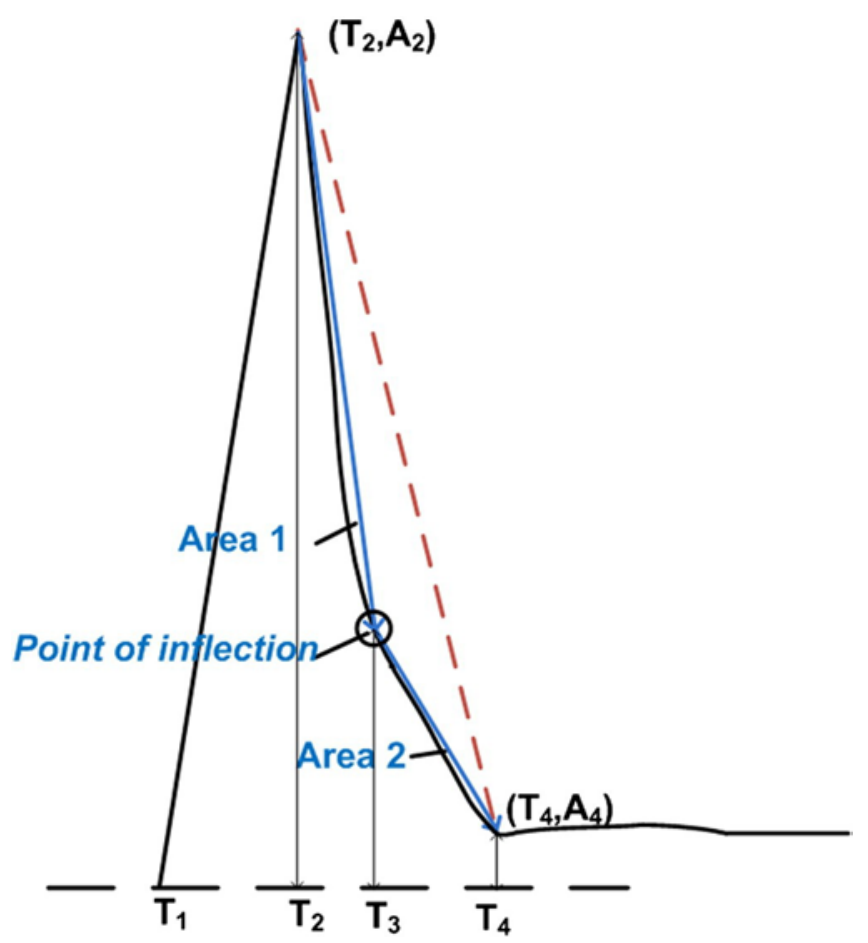

Fig. 2. Finding the point of inflection. The method used is to find the inflection point on the curve which minimises the sum of the areas (area $1+$ area 2 ) between the curve and the two straight lines drawn from the point of inflection - one connecting to the start of the slope $\left(\mathrm{T}_{2}, \mathrm{~A}_{2}\right)$ and the other to the end of the slope $\left(\mathrm{T}_{4}, \mathrm{~A}_{4}\right)$. 


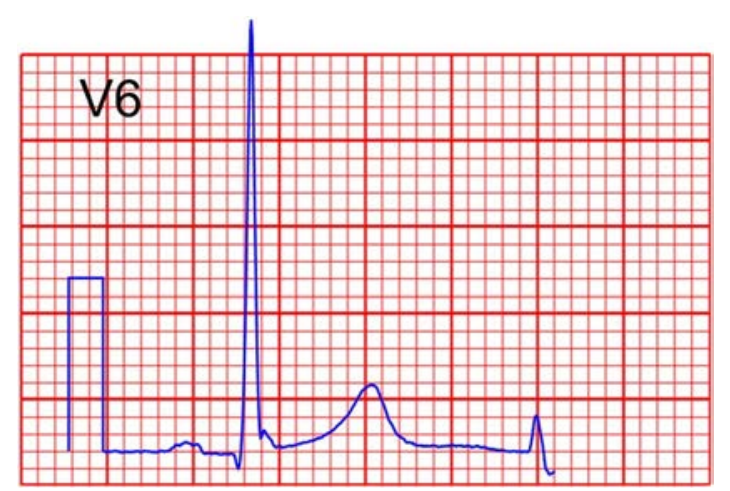

Fig. 3. Example of lead where reviewers disagreed as to whether it was a notch or slur based on a standard printout. Consensus was reached on a slur although an enlarged printout suggested a notch was present.

To further assess the effectiveness of the automated method, it was then carried out on all the ECGs for the dataset of 1496 Caucasian adults and on a further dataset of 1261 apparently healthy Nigerian adults [8]. The ECG recordings on the Nigerian population were made using a Burdick Atria 6100 electrocardiograph using a sampling rate of 500 samples/sec. The prevalence of notching or slurring in the inferolateral leads (I ,II, III, aVL ,aVF ,V4 ,V5 andV6) was found for each set. In addition, where notching or slurring was evident, the prevalence of ST elevation at the QRS end $\geq 0.1 \mathrm{mV}$ in two contiguous leads was determined, either with no restriction on ST slope or with the restriction of ST slope $\leq 10^{\circ}$.

\section{Results}

The two reviewers agreed that there was neither a notch nor a slur in $75 \%$ of the 300 waveforms in the training set, i.e. $50 \times 6$ leads. In $5 \%$ of the leads, one reviewer classified the waveform as having a notch or slur when the other reviewer did not. Of the 60 waveforms which both reviewers classed as ' $\mathrm{N}$ ' or ' $\mathrm{S}$ ', they agreed on the classification in 37 cases, i.e. both classed as a notch or both classed as a slur. An example of a waveform where the reviewers disagreed as to the type is given in Fig. 3.

After revision it was agreed that the training set exhibited a notch in 35/300 leads (11.7\%) and a slur in 28/300 leads (9.3\%).

The automated detection of either a notch or slur had sensitivity (SE) of $92.1 \%$ (58/63), specificity (SP) of $96.6 \%$ (229/237), positive predictive value (PP) of 87.9\% (58/66) and negative predictive value (NP) of 97.9\% (229/234) with respect to the gold standard. For the test set, SE was $90.5 \%$ (38/42), SP was 96.5\% (249/258), PP was 80.1\% (38/47) and
NP was $98.4 \%$ (249/253). Combining the results for the training and test sets, the overall accuracy of the automated method was $95.7 \%$ and the overall precision was $84.9 \%$.

When the automated detection was carried out on the full set of Caucasian adults, $11.5 \%$ of the 11968 inferolateral waveforms were found to have notching or slurring. Evidence of notching or slurring where the amplitude of the notch or slur $\geq 0.1 \mathrm{mV}$ in two contiguous leads was then measured (Table 1).

When the selection was restricted to leads with a horizontal or downsloping ST segment, the total number of cases decreased to 179 (12\%). Of those 179, there were 11 in which notches were detected in two contiguous leads - the remainder were all detected as slurs. With the additional criteria of ST elevation at QRS end being $\geq 0.1 \mathrm{mV}$ and ST slope $\leq 10^{\circ}$, there were only two ECGs which satisfied these criteria. Both ECGs had slurs in the inferior leads.

The method was then used on the dataset of healthy Nigerian adults (consisting of 782 males and 479 females with an age range of 20-87 years). For this dataset, $15 \%$ of the 10088 inferolateral waveforms were identified as having notching/ slurring. The prevalence of notching/slurring in two contiguous inferolateral leads is given in Table 1 . With the added criterion of ST slope $\leq 10^{\circ}$, the total number of cases decreased to 175 (14\%), of which 14 had notches detected in two contiguous leads. One ECG had a notch in leads II and aVF with ST slope $\leq 10^{\circ}$ and ST elevation at QRS end $\geq 0.1 \mathrm{mV}$.

Analysis by age and gender of the presence of notching/ slurring in two contiguous inferolateral leads showed a higher prevalence in males than females in the Caucasian dataset (27\% v 18\%, p b 0.005) (Fig. 4).

The difference with respect to gender was only significant for the under 30 age-group. In the Nigerian dataset, the difference with respect to gender was not significant (30\% vs. $28 \%, p=0.48$ ). There was a decrease in prevalence with age in the male cohort of the Caucasian set. In the Nigerian set, the prevalence was highest in $\leq 29$ years age-group for both males and females.

\section{Discussion}

The automated detection of notching and slurring was highly accurate when compared with the manual detection. There were 16 cases in the combined training and test sets where the reviewers identified the phenomenon as a notch and the program categorised it as a slur. In these cases, the program was failing to categorise the notch as the up and down deflection. This was due either to the notch being of insufficient height or being too close to the end of the $\mathrm{R}$

Table 1

Counts of cases with notching/slurring in two or more contiguous inferolateral leads in three main lead groups i.e. leads II, III, aVF, leads I, aVL and leads V4, $\mathrm{V} 5$, V6, and in combinations of these groups.

\begin{tabular}{|c|c|c|c|c|c|c|c|c|}
\hline & II, aVF, III & $\mathrm{I}, \mathrm{aVL}$ & V4-V6 & I, aVL, V4-V6 & II, III , aVF, V4-V6 & I, aVL, II, III, aVF & I, aVL II, III, aVF, V4-V6 & Total (\% of data set) \\
\hline Caucasian & $193(13 \%)$ & $74(4 \%)$ & $39(3 \%)$ & $11(1 \%)$ & $28(2 \%)$ & $1(0.1 \%)$ & $0(0 \%)$ & $346(23 \%)$ \\
\hline Nigerian & 186 (15\%) & 62 (5\%) & 68 (5\%) & 10 (1\%) & 42 (3\%) & $0(0 \%)$ & $2(0.2 \%)$ & 370 (29\%) \\
\hline
\end{tabular}

For combinations, notching or slurring occurs in two or more contiguous leads in each of the constituent lead groups e.g. for the column headed I, aVL, V4, V5, V6, notching or slurring has been identified in (I and aVL) AND either (V4 and V5) or (V5 and V6). 


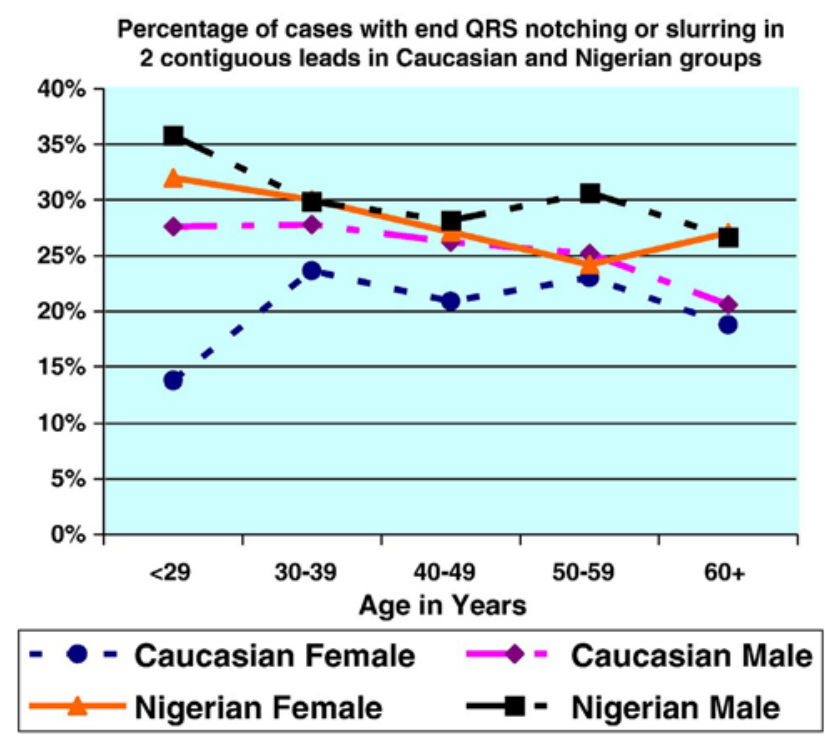

Fig. 4. Comparison of age-related prevalence of ER cases for Caucasian and Nigerian datasets.

wave. However, in these cases, the change of slope met the criteria for a slur. It may be that it is the presence of either form that is important rather than the distinction between the two when considering them as a prognostic aid [1]. On the other hand, in their study, Rollin et al found a higher risk associated with the presence of a notch than a slur [2].

There were cases where it was difficult for the reviewers to decide if the end of the $\mathrm{R}$ wave demonstrated a notch or a slur on account of low amplitude changes, emphasising the need for an internationally agreed definition. Setting criteria for amplitudes and duration may eliminate false reports of notching/slurring.

The prevalence of the ER pattern in two contiguous leads found using the automated method was of the same order of magnitude as that found manually by Sinner et al in the MONICA population [9]. The age and gender analysis of the results for the Caucasian dataset reflected the findings in other studies, e.g. the higher incidence in the young males [4]. The higher incidence rate in the Nigerian dataset reflected the higher prevalence in the black population reported by Kambara and Phillips [10] but was not of the order found for the presence of early repolarisation with or without notching/slurring in an African American population [11]. This is possibly due to the strikingly lower burden of ischaemic heart disease in the Nigerian population, which has been reported to be associated more with ER abnormalities [12]. The insignificant difference in prevalence in the Nigerian dataset with respect to gender may be due to the higher mean BMI for females compared with males in all agegroups [8].

\section{Limitations}

All the ECGs used in the study were recorded at 500 samples/sec. Automated amplitude measurements and changes of slope in the recordings could vary slightly with different sample rates, but 500 samples/sec remains the accepted standard for automated ECG analysis.

The comparison of manual and automated detection was carried out on 600 individual leads from 100 ECGs. This sample set may not have included all possible morphologies of slurred or notched complexes.

The visual grading of the average beats as showing notches or slurs was made using paper printouts. The use of screen displays with zoom capabilities may have given different prevalence.

\section{Conclusions}

It is possible to automate the detection of end QRS notching and slurring with a high degree of accuracy. Once definitions have been agreed, this approach could be used to improve the automated diagnosis of ER, and to help investigations into the prognostic value of ER. Further study is required to measure the racial variation of ER.

\section{References}

[1] Tikkanen JT, Junttila MJ, Anttonen O, Aro AL, Luttinen S, Kerola T, et al. Early repolarization: electrocardiographic phenotypes associated with favorable long-term outcome. Circulation 2011;123:2666-73.

[2] Rollin A, Maury P, Bongard V, Sacher F, Delay M, Duparc A, et al. Prevalence, prognosis, and identification of the malignant form of early repolarization pattern in a population-based study. Am J Cardiol 2012;110(9):1302-8.

[3] Rosso R, Glikson E, Belhassen B, Katz A, Halkin A, Steinvil A, et al. Distinguishing "benign" from "malignant early repolarization": the value of the ST-segment morphology. Heart Rhythm 2012;9:225-9.

[4] Klatsky AL, Oehm R, Cooper RA, Udaltsova N, Armstrong MA. The early repolarization normal variant electrocardiogram: correlates and consequences. Am J Med 2003;115(3):171-7.

[5] The CSE Working Party. Recommendations for measurement standards in quantitative electrocardiography. Eur Heart J 1985;6:815-25.

[6] Junttila MJ, Sager SJ, Tikkanen JT, Anttonen O, Huikuri HV, Myerburg RJ. Clinical significance of variants of J-points and Jwaves: early repolarization patterns and risk. Eur Heart J 2012;33: 2639-43.

[7] Macfarlane PW, Devine B, Clark E. The University of Glasgow (UniG) ECG analysis program. In proceedings of Computing in Cardiology 2005;32:451-4.

[8] Katibi IA, Clark EN, Devine B, Lloyd SM, Macfarlane PW. Normal limits of the electrocardiogram in Nigerians. J Electrocardiol 2013;46: 289.

[9] Sinner MF, Reinhard W, Müller M, Beckmann B-M, Martens E, et al. Association of early repolarization pattern on ECG with risk of cardiac and all-cause mortality: a population-based prospective cohort study (MONICA/KORA). PLoS Med 2010;7(7):e1000314, http:// dx.doi.org/10.1371/journal.pmed.1000314.

[10] Kambara H, Phillips J. Long-term evaluation of early repolarization syndrome (normal variant RS-T segment elevation). Am J Cardiol 1976;38(2):157-61.

[11] Perez MV, Uberoi A, Jain NA, Ashley E, Turakhia MP, Froelicher V. The prognostic value of early repolarization with ST-segment elevation in African Americans. Heart Rhythm 2012;9(4):558-65.

[12] Mukadas AO, Misbau U. Incidence and patterns of cardiovascular disease in north western Nigeria. Nigerian Medical Journal 2009;50(3): $55-7$. 